\title{
Combination of temozolomide and Taxol exerts a synergistic inhibitory effect on Taxol-resistant glioma cells via inhibition of glucose metabolism
}

\author{
DING-GUO GUAN ${ }^{1}$, HAN-MIN CHEN $^{1}$, SHENG-FANG LIAO $^{1}$ and TIAN-ZHI ZHAO ${ }^{2}$ \\ ${ }^{1}$ Department of Neurosurgery, The 180th Hospital of PLA, Quanzhou, Fujian 362000; \\ ${ }^{2}$ Department of Neurosurgery, Affiliated Tangdu Hospital, The Fourth Military Medical University, \\ Xi'an, Shanxi 710038, P.R. China
}

Received March 24, 2014; Accepted June 11, 2015

DOI: $10.3892 / \mathrm{mmr} .2015 .4405$

\begin{abstract}
Malignant gliomas, which comprise the most common type of primary malignant brain tumor, are associated with a poor prognosis and quality of life. Paclitaxel (Taxol) and temozolomide (TMZ) are Food and Drug Administration-approved anticancer agents, which are known to have therapeutic applications in various malignancies. However, similar to other chemotherapeutic agents, the development of resistance to TMZ and Taxol is common. The aim of the present study was to investigate the regulation of glucose metabolism by TMZ and Taxol in glioma cells. The results demonstrated that glioma cells exhibit decreased glucose uptake and lactate production in response to treatment with TMZ; however, glucose metabolism was increased in response to Taxol treatment. Following analysis of TMZ- and Taxol-resistant cell lines, it was reported that glucose metabolism was decreased in the TMZ-resistant cells, but was increased in the Taxol-resistant cells. Notably, a combination of TMZ and Taxol exerted synergistic inhibitory effects on Taxol-resistant glioma cells. However, the synergistic phenotype was not observed following treatment with a combination of 5-fluorouracil and Taxol. Furthermore, restoration of glucose metabolism by overexpression of glucose transporter 1 in Taxol-resistant cells resulted in regained resistance to Taxol. Therefore, the present study proposes a novel mechanism accounting for the synergistic effects of Taxol and TMZ co-treatment, which may contribute to the development of therapeutic strategies for overcoming chemoresistance in patients with cancer.
\end{abstract}

Correspondence to: Dr Ding-Guo Guan, Department of Neurosurgery, The 180th Hospital of PLA, 180 Wai Huayuan Road, Quanzhou, Fujian 362000, P.R. China

E-mail: ggdingguo789@126.com

Key words: temozolomide, Taxol, Taxol-resistant glioma cells, glucose metabolism

\section{Introduction}

Gliomas are primary malignant brain tumors that exhibit aggressive properties and are associated with a poor prognosis in adults $(1,2)$. Glioma cells are able to invade healthy brain tissue and possess enhanced resistance to radiotherapy- and chemotherapy-induced apoptosis (3). Temozolomide (TMZ) comprises an orally bioavailable alkylating agent, which targets nuclear DNA and generates nuclear DNA adducts that block the cell cycle leading to apoptotic cell death (4). Currently, adjuvant chemotherapy with TMZ is the standard treatment for patients exhibiting primary glioblastoma multiforme (GBM) (5,6). The chemotherapeutic regimen is commonly administered as part of the treatment for GBM; however, the majority of patients eventually develop resistance to chemotherapy $(7,8)$. Therefore, understanding the underlying mechanisms that determine tumor response to TMZ is considered to be crucial.

The Warburg effect is defined as the increased utilization of glucose, which is produced by glycolysis (9). Normal tissue relies primarily on mitochondrial oxidative phosphorylation to generate the energy required for cellular processes; however, the majority of cancer cells switch to aerobic glycolysis. This difference suggests that targeting metabolic dependence may be a selective approach for the treatment of clinical patients. Therefore, glycolytic inhibition has been applied as an anticancer strategy in the context of selected components of the glycolytic pathway, including glucose transporters (Gluts), hexokinase, pyruvate kinase M2 and lactate dehydrogenase-A (LDHA) (10). It has previously been reported that LDHA expression and activity are higher in Taxol-resistant breast cancer cells, versus those in parental cells, and downregulation of LDHA resensitized Taxol-resistant cells (11) indicating that dysregulated cellular metabolism may be a therapeutic target for overcoming drug resistance in cancer therapy.

The aim of the present study was to investigate the glucose metabolism of glioma cells in response to treatment with TMZ. The results demonstrated that glioma cells exhibited decreased glucose uptake and lactate production. 


\section{Materials and methods}

Cell culture and antibodies. The U251 and LN229 human glioma cell lines were purchased from the American Type Culture Collection (Manassas, VA, USA). The cells were grown adherently in Dulbecco's modified Eagle's medium (DMEM, Sigma-Aldrich China, Inc., Hong Kong, China) supplemented with $10 \%$ fetal bovine serum (FBS; Sigma-Aldrich China) and $1 \%$ penicillin-streptomycin (Gibco Life Technologies, Grand Island, NY, USA) and maintained in a humidified incubator containing $5 \% \mathrm{CO}_{2}$ at $37^{\circ} \mathrm{C}$. The cells were maintained in these culture conditions for all experiments. Monoclonal antibodies used in the present study were as follows: Anti- $\beta$-actin (cat. no. 4967; Cell Signaling Technology, Inc., Danvers, MA, USA); anti-Glut1 (cat. no. sc-7903; Santa Cruz Biotechnology, Inc., Dallas, TX, USA) and anti-phosphoinositide-dependent kinase-1 (PDK1; cat. no. 3820; Cell Signaling Technology, Inc.). A vector containing Myc-DDK-tagged wild-type Glut1 (cat. no. RC222696), was purchased from OriGene Technologies, Inc., Rockville, MD, USA). Temozolomide (TMZ), 5-fluorouracil (5-FU) and paclitaxel (Taxol) were purchased from Sigma-Aldrich China, Inc.

Generation of Taxol- and TMZ-resistant cell lines. The Taxol- and TMZ-resistant cell lines were generated according to previously described methods $(11,12)$. Briefly, U251 Taxol- or TMZ-resistant cells were developed from parental U251 cells by treating the cells with gradually increasing concentrations $(0.1,0.5,1$ and $2 \mu \mathrm{M})$ of Taxol or TMZ in regular cell culture medium. Taxol- or TMZ-resistant single clones were selected and pooled for the subsequent experiments. All resistant cells were re-selected by treatments every four weeks.

Cell viability assay. The Taxol- and TMZ-resistant cells were treated with the indicated concentrations of Taxol, 5-FU or $\operatorname{TMZ}(0.3,1$ and $0.1 \%$, respectively) for $48 \mathrm{~h}$. The cells were seeded in a 48 -well plate, at a density of $1 \times 10^{4}$ cells/well in $0.2 \mathrm{ml}$ DMEM supplemented with 10\% FBS. Following an overnight incubation under the same culture conditions, each well was refreshed with DMEM for a further $24 \mathrm{~h}$. The cells were then treated with DMEM containing various concentrations of Taxol, TMZ, 5-FU, Taxol + TMZ, or Taxol + 5-FU The therapeutic agent-containing DMEM was refreshed after two days, and the cells were incubated under the same conditions. Finally, cell viability was assessed using an MTT assay (Sigma-Aldrich, St. Louis, MO). Five hours prior to the end of the incubation time, $20 \mu 1$ MTT solution $[5 \mathrm{mg} / \mathrm{ml}$ dissolved in phosphate-buffered saline (PBS)] was added to each well containing cells, followed by incubation at $37^{\circ} \mathrm{C}$ for $5 \mathrm{~h}$. The media were removed by aspiration with a needle and syringe, $200 \mu 1$ dimethylsulfoxide was added to each well and formazan crystals were dissolved by continuous pipetting. Following incubation of the plate at $37^{\circ} \mathrm{C}$ for $5 \mathrm{~min}$, the absorbance was measured at $590 \mathrm{~nm}$ using a plate reader (Multiskan ${ }^{\mathrm{TM}}$ GO; Thermo Fisher Scientific, Waltham, MA, USA). The relative viability was obtained from the absorbance at $590 \mathrm{~nm}$ of therapeutic agent-treated cells divided by the absorbance of untreated cells at $590 \mathrm{~nm}$. The cell viability experiment was repeated three times.
Western blot analysis. The Taxol- and TMZ-resistant cells, as well as Taxol-, 5-FU- or TMZ-treated Taxol- and TMZ-resistant cells were harvested and lysed in a buffer containing $50 \mathrm{mM}$ Tris- $\mathrm{HCl}(\mathrm{pH} 7.5) 150 \mathrm{mM} \mathrm{NaCl}, 2 \mathrm{mM}$ EDTA, $1 \%$ Triton X-100, $1 \mathrm{mM}$ phenylmethylsulfonyl fluoride and Protease Inhibitor Cocktail (Sigma-Aldrich) for $20 \mathrm{~min}$ on ice. Lysates were cleared by centrifugation at $16873 \mathrm{x} \mathrm{g}$ for $10 \mathrm{~min}$ at $4^{\circ} \mathrm{C}$. Supernatants were collected and protein concentrations were determined using the Bradford assay (Bio-Rad Laboratories, Inc., Hercules, CA, USA). The proteins $(50 \mu \mathrm{g})$ were then separated by $10 \%$ SDS-PAGE and transferred to a nitrocellulose membrane (Bio-Rad Laboratories, Inc.). After blocking in PBS containing 5\% non-fat dry milk for $1 \mathrm{~h}$, the membranes were incubated overnight at $4-8^{\circ} \mathrm{C}$ with the primary antibodies diluted in PBS $(1: 5,000)$ with $5 \%$ non-fat dry milk. The membranes were extensively washed with PBS, and incubated at room temperature for $1 \mathrm{~h}$ with horseradish peroxidase-conjugated secondary anti-mouse or anti-rabbit secondary antibodies (1:2,000; cat. no. SA00001-2; Proteintech Group, Inc., Chicago, IL, USA). Following additional washes with PBS, the antigen-antibody complexes were visualized using an enhanced chemiluminescence kit (Pierce Biotechnology, Inc., Rockford, IL, USA).

Glucose uptake assay. U251 cells and TMZ-treated U251 cells were seeded in 12-well plates at a density of $1-3 \times 10^{5}$ cells/well. Culture media was collected at $48 \mathrm{~h}$ and stored at $-20^{\circ} \mathrm{C}$ until assayed. Glucose uptake was measured using an Amplex Red Glucose/Glucose Oxidase Assay kit (Molecular Probes Life Technologies, Carlsbad, CA, USA). Absorbance was measured at $563 \mathrm{~nm}$ using a SpectraMax M5 plate reader (Molecular Devices, Life Technologies, Sunnyvale, CA, USA), and the results were normalized to the quantity of total protein compared with that of the control cells.

Lactate production assay. Lactate production in the cell culture supernatant was detected using a Lactate Assay kit (BioVision, Inc., Milpitas, CA, USA). Results were normalized to the quantity of total protein compared with that of the U251 cells without TMZ treatment.

Plasmid DNA transfection. A vector containing wild-type Glut1 was purchased from OriGene Technologies, Inc. (cat. no. RC222696). Transfection was performed using the Oligofectamine Transfection reagent (Invitrogen Life Technologies, Carlsbad, CA, USA), according to the manufacturer's instructions. Taxol-resistant U251 cells at a density of $10^{6}$ cells/well in $0.2 \mathrm{ml}$ DMEM were transfected with vector $(1: 1,000)$ and Glut1 $(1: 1,000)$. The cells were collected $48 \mathrm{~h}$ post-transfection and prepared for further analysis by western blotting and an MTT assay.

Statistical analysis. An unpaired Student's t-test was used to analyze the data. SPSS version 21.0 (International Business Machines, Armonk, NY, USA) was used for all statistical analyses. All values are expressed as the mean \pm standard error. $\mathrm{P}<0.05$ was considered to indicate a statistically significant difference between values. 
A



B

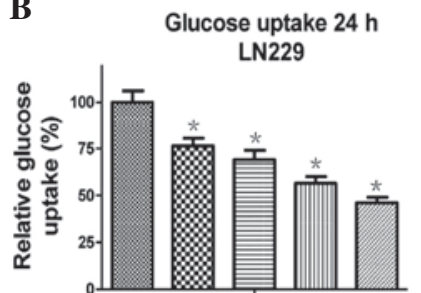

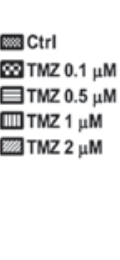
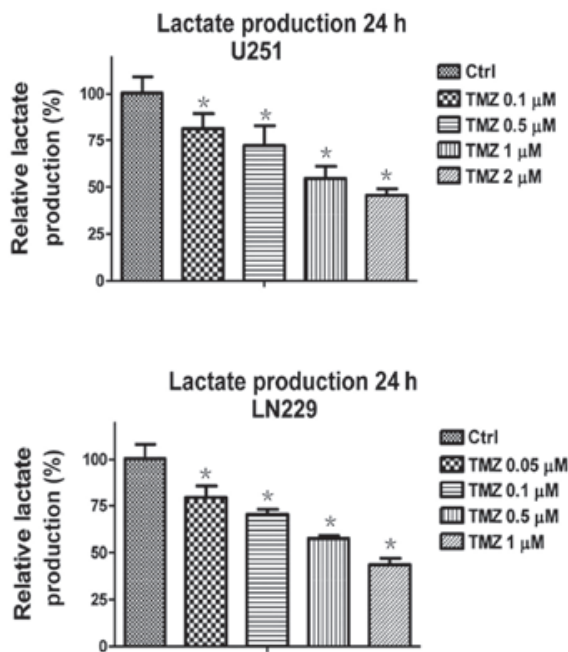

Figure 1. Treatment with TMZ activates glucose metabolism in glioma cells. (A) Glucose uptake (left) and lactate production (right) were measured in U251 cells following treatment with TMZ $(0.1,0.5,1$ and $2 \mu \mathrm{M})$ for $24 \mathrm{~h}$. The relative glucose uptake rates were calculated by comparison with the control cells. (B) Glucose uptake (left) and lactate production (right) were measured in LN229 cells following treatment with TMZ $(0.05,0.1,0.5$ and $1 \mu \mathrm{M})$ for $24 \mathrm{~h}$. The relative glucose uptake rates were calculated by comparison with the control cells. Data are presented as the mean \pm standard error of three independent experiments. TMZ, temozolomide; Ctrl, control.

\section{Results}

Treatment with TMZ decreases the glucose metabolism of glioma cells. A previous study demonstrated that TMZ-resistant glioma cells exhibited elevated mitochondrial function (13), thus suggesting that there is a link between cellular metabolism and TMZ-induced cell apoptosis. Therefore, the present study examined whether treatment of glioma cells with TMZ regulates cellular metabolism. Notably, glucose metabolism was significantly downregulated following treatment with TMZ at various concentrations (Fig. 1). U251 (Fig. 1A) and LN229 (Fig. 1B) cells exhibited decreased glucose uptake and lactate production following treatment with TMZ at low-toxic concentrations for $48 \mathrm{~h}$. These results suggest that TMZ may be administered as an anticancer therapeutic agent through the inhibition of glucose metabolism in glioma cells.

TMZ-resistant glioma cells exhibit decreased glucose metabolism. To investigate the biological significance of TMZ-induced downregulation of glucose metabolism, TMZ-resistant cells were generated using U251 glioma cells, according to the methods of a previous study (12). Briefly, the cells were treated with gradually increasing concentrations of TMZ in cell culture medium and the TMZ-resistant cells were selected (14). After successive treatments for three months, numerous resistant cell clones were developed and pooled for the subsequent experiments. To verify TMZ resistance, the parental cells and resistant pool cells were treated with TMZ at various concentrations for $48 \mathrm{~h}$. As was expected, a cell viability assay demonstrated that TMZ-resistant U251 cells tolerated markedly higher concentrations of TMZ, when compared with TMZ-sensitive cells, which exhibited significant inhibition of viability at 50,100 and $200 \mu \mathrm{M}$ (Fig. 2A). In addition, the glucose uptake and lactate production of the TMZ-resistant U251 cells were decreased (Fig. 2B). In addition, the expression levels of key enzymes associated with glycolysis were detected. Glut1 facilitates the transport of glucose across the plasma membranes of mamma- lian cells, whereas PDK1 inactivates pyruvate dehydrogenase (PDH), which catalyzes the oxidative decarboxylation of pyruvate through the phosphorylation of PDH (15). The present study demonstrated that Glut1 and PDK1 were downregulated in TMZ-resistant cells (Fig. 2C), supporting the results presented in Fig. 2B demonstrating that TMZ-resistant glioma cells exhibit decreased glucose metabolism.

Taxol-resistant glioma cells display upregulated glucose metabolism. The combination of Taxol with numerous anticancer therapeutic agents has been assessed and clinically applied for the treatment of patients with cancer. To investigate the association between dysregulated glucose metabolism and chemosensitivity, a Taxol-resistant cell line was generated from U251 cells. Taxol-resistant U251 cells demonstrated a significant tolerance to Taxol at the doses of $0.5 \mu \mathrm{M}, 1 \mu \mathrm{M}$ and $5 \mu \mathrm{M}$ (Fig. 3A). In addition, glucose uptake and lactate production (Fig. 3B) were detected in Taxol-sensitive and -resistant glioma cells. Taxol-resistant cells exhibited upregulated glucose metabolism, as compared with the Taxol-sensitive cells, suggesting that activated glucose metabolism may be an underlying mechanism of resistance, and may be a therapeutic target for overcoming Taxol resistance in patients with glioma. Concordantly, the expression levels of Glut1 and PDK1 were observed to be upregulated in the Taxol-resistant cells (Fig. 3C).

A combination of Taxol and TMZ exerts a synergistic effect on Taxol-resistant cells via inhibition of glucose metabolism. It has previously been reported that Taxol-resistant breast cancer cells exhibit upregulated glucose metabolism, and treatment with Taxol and glucose metabolism inhibitors demonstrated a greater inhibitory effect on breast cancer (11). The present study observed that treatment with TMZ inhibited glucose metabolism, however Taxol-resistant glioma cells exhibited activated glucose metabolism; therefore, it was hypothesized that a combination of TMZ and Taxol may act synergistically to overcome Taxol resistance via the inhibition of glucose 
$\mathbf{A}$

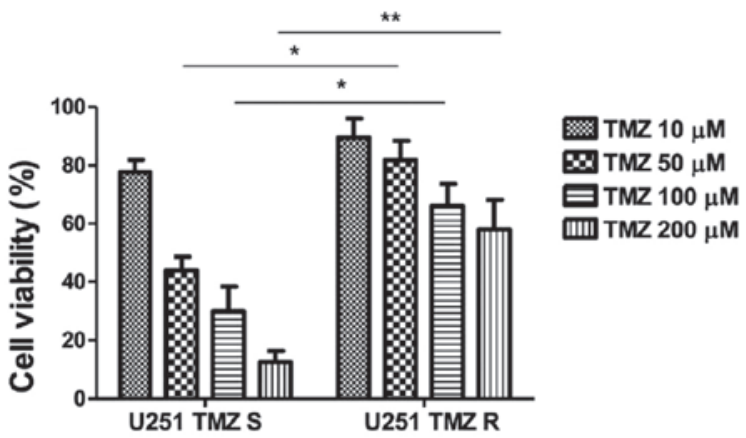

B

\section{Glucose uptake $24 \mathrm{~h}$}

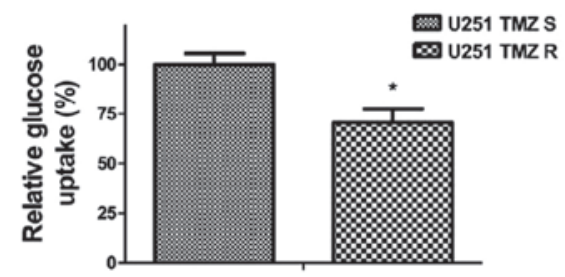

Lactate production $24 \mathrm{~h}$

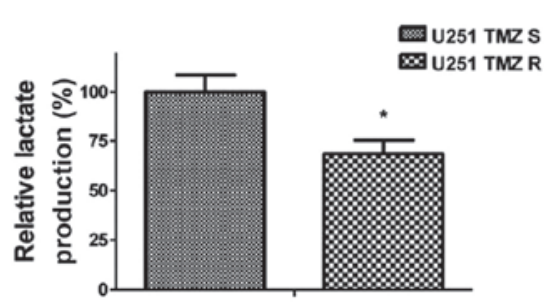

C

U251

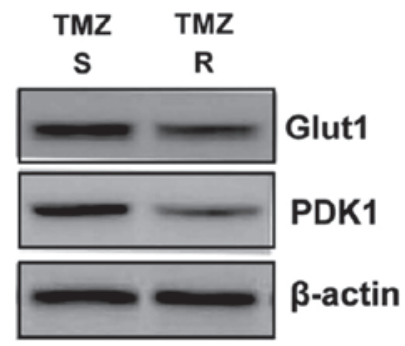

Figure 2. TMZ-resistant cells exhibit downregulated glucose metabolism. (A) Generation of TMZ-resistant cells from U251 glioma cells. Cells were treated with gradually increasing concentrations of TMZ in regular cell culture conditions for the selection of resistant cells. U251 TMZ-resistant clones were pooled and analyzed following treatment with TMZ at the indicated concentrations for $48 \mathrm{~h}$, and cell viability was measured. (B) Glucose uptake (left) and lactate production (right) were measured in U251 TMZ-sensitive and resistant cells. (C) Western blotting demonstrated that the expression of Glut1 and PDK1 were downregulated in TMZ-resistant cells, as compared with the parental cells. $\beta$-actin served as a loading control. Data are presented as the mean \pm standard error of three independent experiments. " $\mathrm{P}<0.05$; ${ }^{* *} \mathrm{P}<0.01$. TMZ, temozolomide; PDK1, phosphoinositide-dependent kinase-1; Glut1, glucose transporter 1; S, sensitive; R, resistant.

A

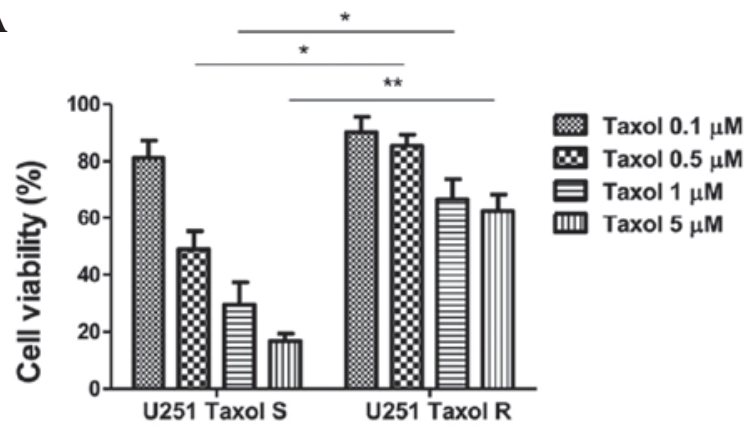

B

Glucose Uptake $24 \mathrm{~h}$

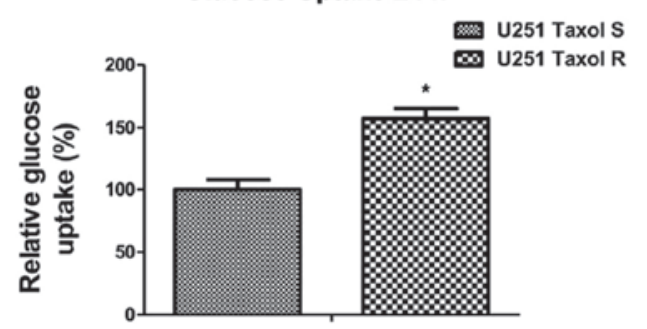

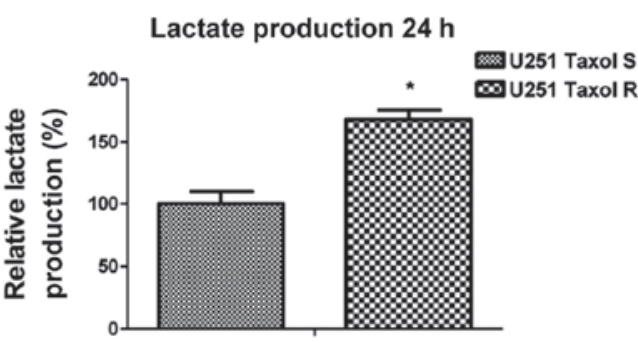

C

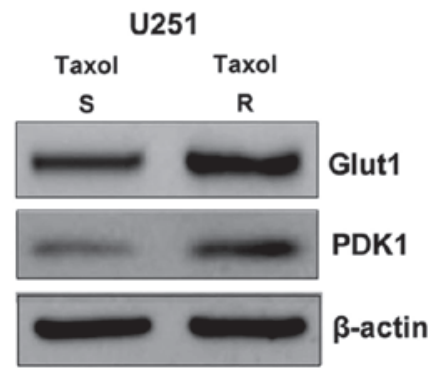

Figure 3. Taxol-resistant cells exhibit upregulated glucose metabolism. (A) Generation of Taxol-resistant cells from U251 glioma cells. Cells were treated with gradually increasing concentrations of Taxol in regular cell culture conditions for the selection of resistant cells. U251 Taxol-resistant clones were pooled and analyzed following treatment with Taxol at the indicated concentrations for $48 \mathrm{~h}$, and cell viability was measured. (B) Glucose uptake (Left) and lactate production (right) were measured in U251 Taxol-sensitive and resistant cells. (C) Western blotting demonstrated that the expression levels of Glut1 and PDK1 were upregulated in Taxol-resistant cells, as compared with the parental cells. $\beta$-actin served as a loading control. Data are presented as the mean \pm standard error of three independent experiments. ${ }^{*} \mathrm{P}<0.05$; ${ }^{* *} \mathrm{P}<0.01$. PDK1, phosphoinositide-dependent kinase-1; Glut1, glucose transporter 1; S, sensitive; R, resistant.

metabolism. Experiments were designed to examine whether treating Taxol-resistant glioma cells with TMZ would result in synergistic therapeutic effects. As shown in Fig. 4A, no significant inhibitory effects were observed in the Taxol-resistant cells treated with Taxol or TMZ alone; however, administering a combination of Taxol and TMZ resulted in significant inhibition of cell viability. It has previously been reported that treatment with 5-FU may induce glucose metabolism in colon cancer cells and has similar regulatory effects on glucose metabolism as Taxol (15). Therefore, in the present study, resistant cells 
A

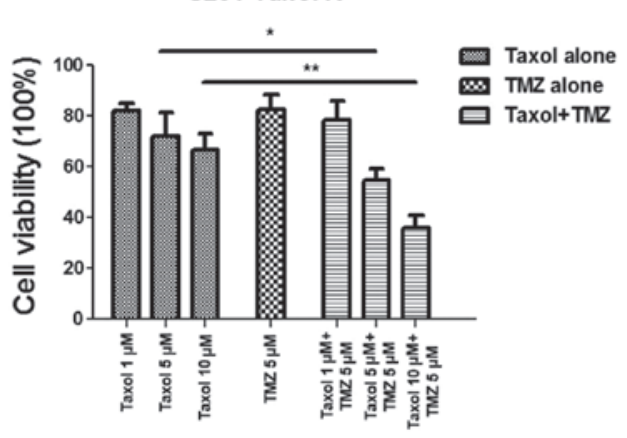

B

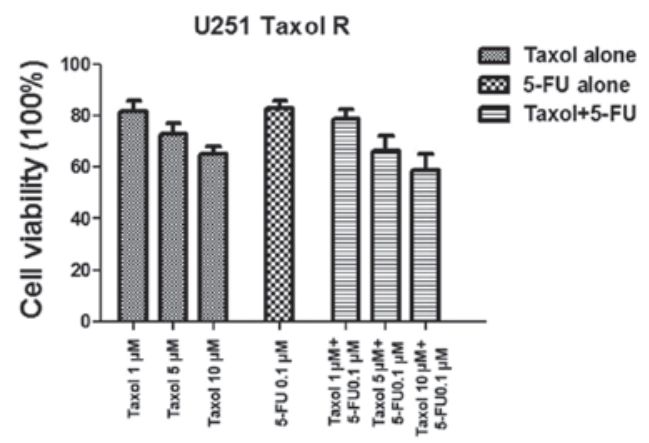

Figure 4. A combination of Taxol and TMZ, but not Taxol and 5-FU, exerts synergistic effects on Taxol-resistant cells. (A) Taxol-resistant U251 glioma cells were treated with Taxol alone at doses of 1,5 and $10 \mu \mathrm{M}$; TMZ alone at $5 \mu \mathrm{M}$; or Taxol + TMZ for $48 \mathrm{~h}$, following which cell viability was measured. (B) Taxol-resistant U251 cells were treated with Taxol alone at 1,5 and $10 \mu \mathrm{M} ; 5-\mathrm{FU}$ alone at $0.1 \mu \mathrm{M}$; or Taxol +5 -FU for $48 \mathrm{~h}$, following which cell viability was measured. Data are presented as the mean \pm standard error of three independent experiments. ${ }^{*} \mathrm{P}<0.05 ;{ }^{* *} \mathrm{P}<0.01$. TMZ, temozolomide; R, resistant; 5 -FU, 5 -fluorouracil.

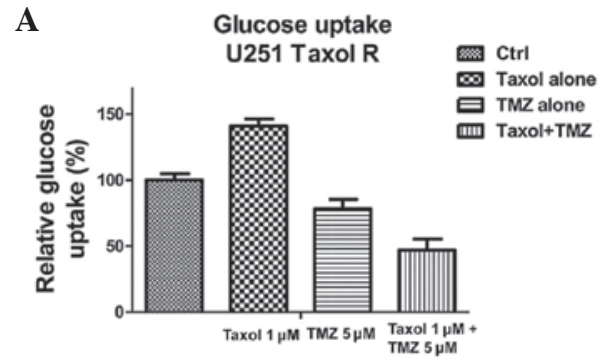

B

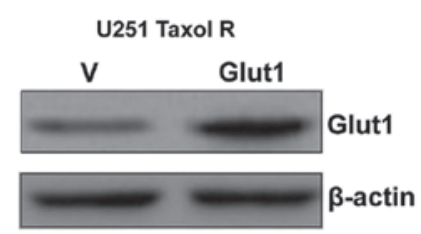

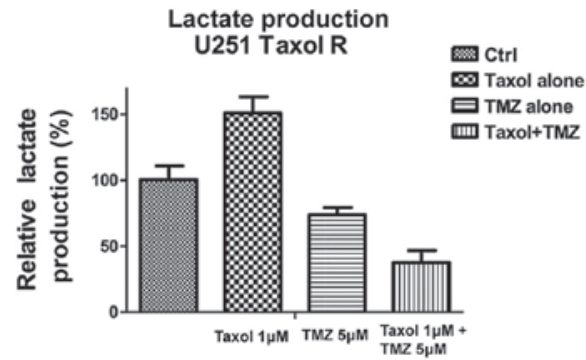

U251 Taxol R

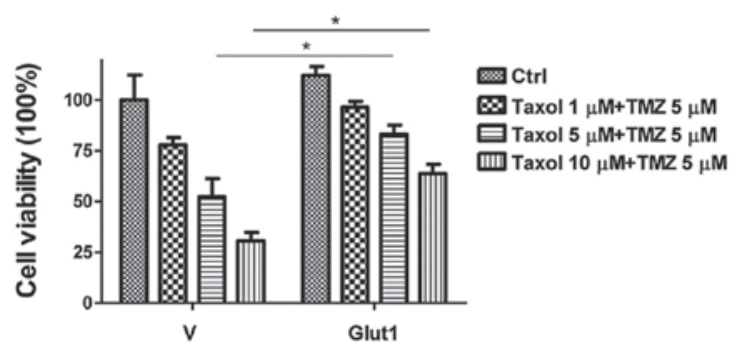

Figure 5. Overexpression of Glut1 renders Taxol-resistant cells unsusceptible to Taxol and TMZ combined treatment. (A) Treatment with a combination of Taxol and TMZ inhibited glucose uptake (left) and lactate production (right). Taxol-resistant U251 glioma cells were treated with Taxol alone at $1 \mu \mathrm{M}$; TMZ alone at $5 \mu \mathrm{M}$, or Taxol + TMZ for $48 \mathrm{~h}$, following which glucose uptake (left) and lactate production (right) were measured. (B) Western blotting demonstrated that the expression levels of Glut1 in U251 Taxol-resistant cells transiently transfected with Glut1 were higher, as compared with cells transfected with a vector control (left). U251 Taxol-resistant cells were transiently transfected with vector control or wild-type Glut 1 for $48 \mathrm{~h}$, and were plated into 48 -well plates for the indicated treatments for $48 \mathrm{~h}$, followed by measurements of cell viability. $\beta$-actin served as a loading control. Data are presented as the mean \pm standard error of three independent experiments. " $\mathrm{P}<0.05$. TMZ, temozolomide; Ctrl, control; R, resistant; Glut1, glucose transporter 1; V, vector.

were treated with a combination of Taxol and 5-FU; however, no improvement in the chemotherapeutic effects was noted (Fig. 4B), indicating that the synergistic inhibitory effects exerted by the combination of Taxol and TMZ may be due to the inhibition of glucose metabolism.

To support this conclusion, the glucose uptake and lactate production of the cells were determined following the combined treatment (Fig. 5A). The glucose uptake and lactate production were significantly decreased following treatment with a combination of Taxol and TMZ. To further verify the hypothesis, an expression vector containing Glut1 was transiently transfected into the Taxol-resistant U251 cells (Fig. 5B). Restoration of Glut1 expression recovered the glucose metabolism in U251 Taxol-resistant cells (data not shown), and rendered cells resistant to the combined treatment (Fig. 5B). These data indicate that the inhibition of glucose metabolism may specifically account for the synergistic effects of Taxol and TMZ combined treatment in Taxol-resistant glioma cells.

\section{Discussion}

Paclitaxel (Taxol) is one of numerous tumoricidal agents that target microtubules, and is widely administered as a chemotherapeutic agent in the treatment of various types of human cancer (16). The primary cellular targets of Taxol are cancer cell microtubules, which are vital for mitotic activity, cellular motility and proliferative capacity (17). However, despite an impressive initial clinical response, the majority of patients eventually develop resistance to Taxol. Therefore, the ability to reduce chemoresistance is particularly important for patients with cancer. One known mechanism associated with cancer cell resistance to Taxol, and other microtubule-stabilizing agents, 
is the high expression of the membrane, P-glycoprotein, which functions as a drug-efflux pump (18). Other cellular mechanisms include cell cycle deregulation $(19,20)$ and alterations to tubulin structure $(21,22)$. A recent study reported that Taxol resistance in breast cancer cells was mediated by the hippo signaling pathway (23). In addition, a correlation between Aurora kinase A and Taxol resistance has been detected in a xenograft model of breast cancer (24). However, the detailed mechanisms for the development of Taxol resistance in glioma cells remain to be elucidated.

Cancer cells use aerobic glycolysis, with reduced mitochondrial oxidative phosphorylation, to metabolize glucose, whereas healthy cells rely on oxidative phosphorylation. Therefore, the metabolic dependencies of cancer cells may be exploited for cancer treatment via inhibition of metabolic enzymes, in order to improve the efficacy of cancer therapy and overcome therapeutic resistance. It has been reported that numerous anticancer agents may be combined with glycolysis inhibitors, such as 5-FU (25), cisplatin (26), daunorubicin (27), Taxol (28), trastuzumab (29) and doxorubicin (30), in order to exert a synergistic inhibitory effect on cancer cells. In addition, the inhibition of glucose uptake has been shown to sensitize cancer cells to daunorubicin, and resensitize drug-resistant cells (27). A previous study demonstrated that targeting dysregulated glucose metabolism in breast cancer cells led to reduced trastuzumab resistance (29). Furthermore, in vivo experiments demonstrated that 2-deoxy-D-glucose increased the efficacy of adriamycin and paclitaxel in human osteosarcoma and non-small cell lung cancers (31). However, the detailed mechanisms for the efficacy of combining chemotherapeutic agents with glycolysis inhibitors remains unclear.

The present study reported that a combination treatment with TMZ and Taxol sensitized Taxol-resistant glioma cells via the inhibition of glucose metabolism. Significant inhibition of glucose metabolism was observed in the TMZ-resistant cells, whereas increased glucose metabolism was observed in the Taxol-resistant cells. This phenotype triggered the investigation of the synergistic effects of a combination of these two chemotherapeutic agents. It has been reported that inhibition of glucose metabolism by oxamate resensitizes Taxol-resistant breast cancer cells to Taxol (11). Since TMZ was able to inhibit glucose metabolism, the present results demonstrate that treatment with TMZ enhances the cytotoxic effects on Taxol-resistant glioma cells by inhibiting glucose metabolism. A previous study reported that 5-FU treatment in cancer cells activated glucose uptake and lactate production (15). Conversely, in the present study, as compared with the combination of TMZ and Taxol, 5-FU treatment did not exert an improved inhibitory effect on glioma cells when combined with Taxol, thus suggesting that the inhibition of glucose metabolism may be the mechanism for the synergistic effect of TMZ and Taxol on Taxol-resistant cells. In addition, recovering the glycolysis signaling pathway by exogenous overexpression of Glut1 facilitated glioma cells with obtaining resistance to Taxol, indicating that dysregulated glucose metabolism may be the underlying mechanism of the synergistic effects of TMZ and Taxol. However, the detailed mechanisms are currently being investigated. The aim of future studies will be to screen additional chemotherapeutic agents and assess increasingly efficient chemotherapeutic strategies, and in vivo experiments will be performed to verify the results of animal models.

In conclusion, the present study provides a novel perspective on the synergistic effects of combined chemotherapeutic agents, and contributes to the development of clinical strategies for the treatment of patients with glioma.

\section{References}

1. Huse JT, Holland E and DeAngelis LM: Glioblastoma: Molecular analysis and clinical implications. Annu Rev Med 64: 59-70, 2013.

2. Tanase CP, Enciu AM, Mihai S, Neagu AI, Calenic B and Cruceru ML: Anti-cancer therapies in high grade gliomas. Curr Proteomics 10: 246-260, 2013.

3. Omuro A and DeAngelis LM: Glioblastoma and other malignant gliomas: A clinical review. JAMA 310: 1842-1850, 2013.

4. Thomas RP, Recht L and Nagpal S: Advances in the management of glioblastoma: The role of temozolomide and MGMT testing. Clin Pharmacol 5: 1-9, 2013.

5. Fiorentino A, Chiumento C, Caivano R, Cozzolino M, Pedicini P and Fusco V: Adjuvant radiochemotherapy in the elderly affected by glioblastoma: Single-institution experience and literature review. Radiol Med 118: 870-881, 2013 (In Italian).

6. Sengupta S, Marrinan J, Frishman C and Sampath P: Impact of temozolomide on immune response during malignant glioma chemotherapy. Clin Dev Immunol 2012: 831090, 2012.

7. Chamberlain MC: Temozolomide: Therapeutic limitations in the treatment of adult high-grade gliomas. Expert Rev Neurother 10: 1537-1544, 2010.

8. Jiang G, Wei ZP, Pei DS, Xin Y, Liu YQ and Zheng JN: A novel approach to overcome temozolomide resistance in glioma and melanoma: Inactivation of MGMT by gene therapy. Biochem Biophys Res Commun 406: 311-314, 2011.

9. Vander Heiden MG, Cantley LC and Thompson CB: Understanding the Warburg effect: The metabolic requirements of cell proliferation. Science 324: 1029-1033, 2009.

10. Zhao Y, Butler EB and Tan M: Targeting cellular metabolism to improve cancer therapeutics. Cell Death Dis 4: e532, 2013.

11. Zhou M, Zhao Y, Ding Y, Liu H, Liu Z, Fodstad O, Riker AI, Kamarajugadda S, Lu J, Owen LB, et al: Warburg effect in chemosensitivity: Targeting lactate dehydrogenase-A re-sensitizes taxol-resistant cancer cells to taxol. Mol Cancer 9: 33, 2010.

12. Pan Q, Yang XJ, Wang HM, Dong XT, Wang W, Li Y and Li JM: Chemoresistance to temozolomide in human glioma cell line U251 is associated with increased activity of O6-methylguanine-DNA methyltransferase and can be overcome by metronomic temozolomide regimen. Cell Biochem Biophys 62: 185-191, 2012.

13. Oliva CR, Moellering DR, Gillespie GY and Griguer CE: Acquisition of chemoresistance in gliomas is associated with increased mitochondrial coupling and decreased ROS production. PLoS One 6: e24665, 2011.

14. Fujiwara S, Kawano Y, Yuki H, Okuno Y, Nosaka K, Mitsuya H and Hata H: PDK1 inhibition is a novel therapeutic target in multiple myeloma. Br J Cancer 108: 170-178, 2013.

15. Shin YK, Yoo BC, Hong YS, Chang HJ, Jung KH, Jeong SY and Park JG: Upregulation of glycolytic enzymes in proteins secreted from human colon cancer cells with 5-fluorouracil resistance. Electrophoresis 30: 2182-2192, 2009.

16. Yusuf RZ, Duan Z, Lamendola DE, Penson RT and Seiden MV: Paclitaxel resistance: Molecular mechanisms and pharmacologic manipulation. Curr Cancer Drug Targets 3: 1-19, 2003.

17. Orr GA, Verdier-Pinard P, McDaid H and Horwitz SB: Mechanisms of Taxol resistance related to microtubules. Oncogene 22: 7280-7295, 2003.

18. Donnenberg VS and Donnenberg AD: Multiple drug resistance in cancer revisited: The cancer stem cell hypothesis. J Clin Pharmacol 45: 872-877, 2005.

19. Kavallaris M, Kuo DY, Burkhart CA, Regl DL, Norris MD, Haber M and Horwitz SB: Taxol-resistant epithelial ovarian tumors are associated with altered expression of specific beta-tubulin isotypes. J Clin Invest 100: 1282-1293, 1997.

20. Tan M, Jing T, Lan KH, Neal CL, Li P, Lee S, Fang D, Nagata Y, Liu J, Arlinghaus R, et al: Phosphorylation on tyrosine-15 of p34(Cdc2) by ErbB2 inhibits p34(Cdc2) activation and is involved in resistance to taxol-induced apoptosis. Mol Cell 9: 993-1004, 2002. 
21. Martello LA, Verdier-Pinard P, Shen HJ, He L, Torres K, Orr GA and Horwitz SB: Elevated levels of microtubule destabilizing factors in a Taxol-resistant/dependent A549 cell line with an alpha-tubulin mutation. Cancer Res 63: 1207-1213, 2003.

22. Panda D, Miller HP, Banerjee A, Ludueña RF and Wilson L: Microtubule dynamics in vitro are regulated by the tubulin isotype composition. Proc Natl Acad Sci USA 91: 11358-11362, 1994.

23. Lai D, Ho KC, Hao Y and Yang X: Taxol resistance in breast cancer cells is mediated by the hippo pathway component TAZ and its downstream transcriptional targets Cyr61 and CTGF. Cancer Res 71: 2728-2738, 2011.

24. Li Y, Tang K, Zhang H, Zhang Y, Zhou W and Chen X: Function of Aurora kinase A in Taxol-resistant breast cancer and its correlation with P-gp. Mol Med Rep 4: 739-746, 2011.

25. Tong J, Xie G, He J, Li J, Pan F and Liang H: Synergistic antitumor effect of dichloroacetate in combination with 5-fluorouracil in colorectal cancer. J Biomed Biotechnol 2011: 740564, 2011.

26. Guo W, Zhang Y, Chen T, Wang Y, Xue J, Zhang Y, Xiao W, Mo X and Lu Y: Efficacy of RNAi targeting of pyruvate kinase M2 combined with cisplatin in a lung cancer model. J Cancer Res Clin Oncol 137: 65-72, 2011.
27. Cao X, Fang L, Gibbs S, Huang Y, Dai Z, Wen P, Zheng X, Sadee W and Sun D: Glucose uptake inhibitor sensitizes cancer cells to daunorubicin and overcomes drug resistance in hypoxia. Cancer Chemother Pharmacol 59: 495-505, 2007.

28. Liu Y, Cao Y, Zhang W, Bergmeier S, Qian Y, Akbar H, Colvin R, Ding J, Tong L, Wu S, et al: A small-molecule inhibitor of glucose transporter 1 downregulates glycolysis, induces cell-cycle arrest, and inhibits cancer cell growth in vitro and in vivo. Mol Cancer Ther 11: 1672-1682, 2012.

29. Zhao Y, Liu H, Liu Z, Ding Y, Ledoux SP, Wilson GL, Voellmy R, Lin Y, Lin W, Nahta R, et al: Overcoming trastuzumab resistance in breast cancer by targeting dysregulated glucose metabolism. Cancer Res 71: 4585-4597, 2011.

30. Nakano A, Tsuji D, Miki H, Cui Q, El Sayed SM, Ikegame A, Oda A, Amou H, Nakamura S, Harada T, et al: Glycolysis inhibition inactivates $\mathrm{ABC}$ transporters to restore drug sensitivity in malignant cells. PLoS One 6: e27222, 2011.

31. Hadzic T, Aykin-Burns N, Zhu Y, Coleman MC, Leick K, Jacobson GM and Spitz DR: Paclitaxel combined with inhibitors of glucose and hydroperoxide metabolism enhances breast cancer cell killing via $\mathrm{H}_{2} \mathrm{O}_{2}$-mediated oxidative stress. Free Radic Biol Med 48: 1024-1033, 2010. 